\title{
Spatiotemporal Transport Processes in Semiconductor Gas Discharge Structure with GaAs Photodetector
}

\author{
H. YÜCEL KURT ${ }^{a, *}$ AND B.G. SALAMOV ${ }^{a, b}$ \\ ${ }^{a}$ Department of Physics, Faculty of Arts and Sciences \\ Gazi University, 06500 Teknikokullar, Turkey \\ ${ }^{b}$ Institute of Physics, National Academy of Science \\ Baku 370143, Azerbaijan \\ (Received September 7, 2007; revised version April 15, 2008; \\ in final form May 7, 2008)
}

\begin{abstract}
In a semiconductor gas discharge structure with diameters much larger than an inter-electrode distance, the effects of different parameters (i.e. electrode separation, gas pressure, diameter of the GaAs photodetector, etc.) on electrical breakdown and current oscillations were studied. Non-stationary and non-homogeneous states are generated in the structure, through the spatially uniform irradiation of the semiconductor photodetector. Instabilities occur due to the nonlinear features of the semiconductor photocathode, while the gas discharge serves to visualize transport processes in GaAs. Spatiotemporal variations of current and discharge light emissions are studied with the above-mentioned control parameters. Transformation of the profile and amplitude of the current densities of the filaments in different regions of the current-voltage characteristic are widely studied. Instabilities of spatially non-uniform distributions resulting in the formation of multiple current filaments with increasing voltages above the critical values are observed. A semiconductor gas discharge structure with an $N$-shaped current-voltage characteristic is analyzed via both the current and discharge light emissions data which shows the electrical instability in the GaAs photodetector.
\end{abstract}

PACS numbers: 71.55.Eq, 72.20.Ht

\section{Introduction}

The planar dc-driven gas discharge system with a high resistivity GaAs cathode has found numerous applications in the past decade, especially in the registration and visualization of infrared (IR) images [1-3]. In such gas discharge devices

*corresponding author; e-mail: hkurt@gazi.edu.tr or egokcen52tr@hotmail.com 
with a small active volume, the role of the space charge of the ions in the discharge process is diminished, and the transport of ionized particles in the gap is mainly controlled by the electric field, determined by surface charges on electrodes (see, e.g. [4]). Electronic phenomena occurring at a contact between a semiconductor and gas discharge plasma has a number of special features and has been among the least investigated semiconductor contact phenomena. Penetration of a double electrical layer charge into the interior of a semiconductor results in a strong dependence of the value and spatial distribution of the current density on the state of the semiconductor and makes it possible to control both the current and the radiation emitted by the gas discharge. Many technical devices operate in nonlinear regimes. Among them are lasers [5] and different semiconductor devices [6, 7]. Many applications e.g. pulse lasers in technology and optical communication, requires a stable and controllable operation of devices. In this manner, Lippi et al. [8] have suggested a method for controlling gas discharge $\mathrm{CO}_{2}$ and semiconductor lasers to suppress oscillations in the emitted power of transient modes. Compared with high-power discharge systems (e.g. arc discharge devices) microdischarge devices are characterized by a small characteristic dimension of the discharge volume, by a low density of charge carriers in the discharge gap, and, as a consequence, by a relatively low electrical power dissipated in a device. At the same time, semiconductor gas discharge structure (SGDS) uses microdischarges occurring in small volume cells that involve various materials and complicated structures, the discharge characteristics strongly depend on the cell geometry.

Presently, there exists a special interest in semi-insulating (SI) GaAs crystal properties as such crystals become promising for radiation detector applications [9] and SI GaAs has an increasing relevance, e.g., as a particle detector [10, 11] and for optical data storage. The study of instabilities in semiconductors takes intense interest and most of the instabilities display spatiotemporal patterns. Electrical non-linearities in semiconductors are good examples of non-integrable systems since they present a rich variety of complex behavior. Usually the complex phenomena in the devices occurs under sufficiently high-excitation conditions such as large electric or magnetic fields, strong optical irradiation, or high-current injection [12]. The theory [12] and experiment [13] shows that the current flowing through a semiconductor can oscillate if a sufficiently high dc bias is applied to the sample. These oscillations are caused by domains of high electric field traveling from cathode to anode [14]. In this context, Rajbenbach et al. [15] demonstrated a straightforward electrooptic technique for the visualization of electrical domains in SI GaAs:Cr which was based on using the crystal as the active component of a transverse electrooptic two-dimensional light modulator. They were subsequently able to measure the domain velocity as functions of applied voltage and illumination. Besides, they observed stationary gratings by applying ac sawtooth voltages. The pitch of the gratings could then be controlled by varying the frequency of ac voltage. In a different study, Ellin et al. [13] investigated electrical domains in SI 
GaAs:Cr following the method of Rajbenbach et al. and demonstrated how the domain movement can be optically controlled.

In one of our previous studies [16], we have found that the electrical instability in the SGDS occurs due to the nonlinearity of the semiconductor electrode, while the gas discharge serves to visualize transport processes in GaAs. We have seen that high-voltages application to such a system gives rise to non-uniform spatial distribution of the current disturbing the operation of the device. The aim of present study is to shed a light on some new results relating spatiotemporal transport behavior in SI GaAs photodetector and to understand the physical processes involved in current instabilities occurring on photodetectors in a wide region of gas pressures $p$ (28-550 Torr), interelectrode distances $d(45-525 \mu \mathrm{m})$ and different diameters $D(5-22 \mathrm{~mm})$. At the same time, this paper quantitatively investigates the spatial distribution of the current density formed as a result of loss of stability of a homogeneous current state in a high-resistivity GaAs photodetector subjected to strong electric fields.

\section{Experimental procedure}

In this study, we compare transport properties of structures of the two types, one of which contains a SGDS with a SI GaAs photodetector [17]. Both structures (see Fig. 1a and b) sequentially operate with the same GaAs photodetector. Main two parts of the SGDS include a photodetector and a gas layer which specifies the basic properties of the system. A gas layer is sandwiched between the glass plate and the semiconductor plate in Fig. 1b. It is known [18] that if one of the electrodes has a form of semiconductor plate in a plane gas discharge cell, then a gas discharge current in the cell is distributed over the entire area of electrodes causing a uniform discharge light emission (DLE). When the current passes through such a structure, the spatial distribution of the DLE intensity in the gap reproduces the distribution of the conductivity of the IR photodetector. In the experiments, a Cr-doped SI GaAs [19], $n$-type high resistivity $\left(\rho \approx 10^{8} \Omega \mathrm{cm}\right)$ plate with $(100)$ orientation in the plane of natural growth of the crystal, is used as the semiconducting photodetector. The diameter and the thickness of the GaAs photodetector are $50 \mathrm{~mm}$ and $1 \mathrm{~mm}$, respectively. On the illuminated side of the photodetector, a conducting vacuum-evaporated Ni-layer with approximately $40 \mathrm{~nm}$ thickness is coated.

The anode is a disk of glass (with $50 \mathrm{~mm}$ diameter and $2 \mathrm{~mm}$ thickness) coated with a thin layer of a transparent conductor $\mathrm{SnO}_{2}$. The sheet resistance of the $\mathrm{SnO}_{2}$ layer is at the range of 15 and $20 \Omega / \square$ and this value is of the order of $10 \Omega / \square$ for Ni film. The resistances are negligible when they are compared with the sheet resistance of the GaAs layer, which is $7.3 \times 10^{8} \Omega / \square$ in the nonirradiated case. The discharge gap between the glass plate and the photodetector is filled with atmospheric room air. By applying a high voltage $V_{0}$ between Ni contact and $\mathrm{SnO}_{2}$ layer, a discharge is ignited in the gap. This corresponds to a discharge 


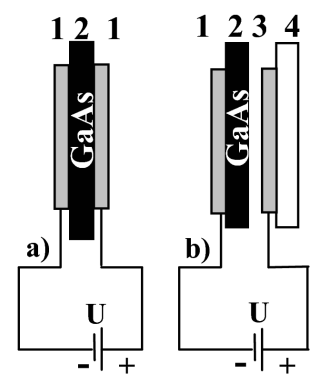

Fig. 1. Investigated structure (a) in the absence and (b) in the presence of a gas discharge gap: 1 - Ni contact; 2 - GaAs photodetector; 3 - gas discharge gap; 4 anode.

operation in the Townsend regime (this stable form of discharge is employed in spectral image converters [17, 20] with photosensitive GaAs detector possessing linear current-voltage characteristic (CVC)). The surface of the semiconductor cathode is separated from a flat anode by an insulating mica sheet with a circular aperture at its center. Changeable thickness of the insulator makes it possible to vary the size of the interelectrode air gap, $d$, between 45 and $525 \mu \mathrm{m}$.

Typical diameters of the active electrode areas, $D$ (i.e. gas discharge gap or diameters of the circular through-aperture in the insulator) are 5, 9, 12, 18 and $22 \mathrm{~mm}$. The CVCs are recorded gradually by varying the voltage with a rate of $5 \mathrm{~V} \mathrm{~s}^{-1}$. For further details regarding to the experimental setup, we refer to [21]. A simple optical arrangement is employed to ensure spatial homogeneous illumination on the photocathode. The illumination intensity is varied at the range of $10^{-6}-10^{-2} \mathrm{~W} \mathrm{~cm}^{-2}$ by the use of filters. The maximum illumination intensity is found around $4 \times 10^{-2} \mathrm{~W} \mathrm{~cm}^{-2}$. The semiconductor is photosensitive up to a wavelength of $0.89 \mu \mathrm{m}$. In the IR region the photoconductivity in GaAs is attributed to $\mathrm{Cr}$ impurities. The IR radiation excites the photosensitive detector of the cell thus controls the current density and DLE from the gas discharge gap.

A computer with appropriate and interface units are used to digitize the CVC measurements. The current through the cell and the voltage drop between the electrodes are recorded simultaneously by using a digital multimeter (Keithley 199) and the high-voltage power supply (Stanford PS 325, 2500 V-25 W). The DLE from the cell is measured using a computerized photon counting unit, actually the photon counting head of a commercial optically stimulated luminescence dating equipment (ELSEC 9010-Littlemore Scientific Eng.). The photomultiplier tube of the unit (Thorn-EMI 9235 QA) has a high sensitivity in the UV-blue region of the spectrum, which coincides with the emission from the cell. In order to reject the IR leakage from the system a glass filter mainly transmitting in 330-700 nm (Schott BG39) was used. The SGDS cell includes glass/quartz window to allow optical access to the sustained discharge. The working pressure is adjusted by a 
needle valve placed between the cell and the pump system. The DLE is recorded through a transparent anode with a CCD camera.

Before coming to our results, it is necessary to discuss briefly the mechanism governing domain formation. In our material, the trapping of conduction-band electrons by deep level impurity sites are considered to increase with electric field. The material will then exhibit a negative differential conductivity (NDC) and will consequently be inherently unstable: a local increase in electric field (e.g., due to random fluctuation) will decrease the local concentration of free carriers, thereby increasing the local (and total) resistance. This in turn will lead to a local increase in the electric field, and so on. We note that while a domain is propagating inside the crystal, the dc current through the crystal will be lower than when in the absence of a domain. When a dc voltage, exceeding a certain threshold value $V_{\text {cr }}$, is applied to the crystal one observes as stated Ref. [15] the domains of high field propagated across the crystal from cathode to anode, with a velocity which increased with increasing voltage and intensity of IR illumination. In addition, we note that the formation or "nucleation" of the domains at the cathode is significantly facilitated by displacing the input beam of the IR illumination, slightly towards the cathode, thereby altering the intensity distribution across the crystal. This changed distribution leads to a reduction of the threshold voltage $V_{\text {cr }}$ (see Fig. 2).
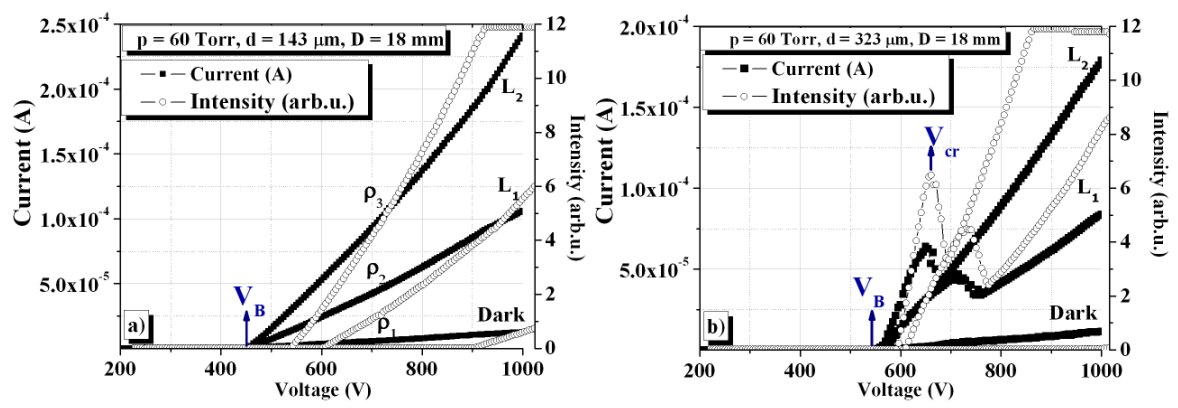

Fig. 2. CVCs of a planar discharge cell in darkness and under weak and maximum illumination intensities of light $L_{1}$ and $L_{2}$, respectively: (a) the curves $\rho_{1}-\rho_{3}$ represent the CVC for three resistivities of the SI GaAs photodetector for $d=143 \mu \mathrm{m}$; (b) CVCs with $N$-type NDC of the SI GaAs photodetector for electrode separation $d=323 \mu \mathrm{m}$.

\section{Results and discussions}

In order to describe the dynamic properties of SGDS, it is convenient to use transient characteristics measured in a system that turns from a state with a low density of electric current into a state with much larger current density. In discussing these experimental results we should initially determine which type of discharge occurs in the SGDS with the semiconductor cathode. Under dc conditions we deal with a self-sustained Townsend discharge, which is deduced, in 
particular, from the range of the current densities. In what follows, we will consider a dynamic theory based on the ordinary electron avalanche multiplication mechanism in a gas and on stripping of electrons from the cathode as a result of ion emission and the photoelectric effect. We assume that the current density is low and that the electric field perturbation produced by the space charge is weak, so that perturbation theory is applicable [22]. The dynamical processes in the structure can be interpreted by using the following pair of coupled differential equations for the electric field $E$ and the concentration of free carriers $N$ in the gap:

$$
\begin{aligned}
& \mathrm{d} E / \mathrm{d} t=\left(1 / \tau_{\mathrm{M}}\right)\left(E_{\mathrm{M}}-E\right)-b N E, \\
& \mathrm{~d} N / \mathrm{d} t=\left(N / \tau_{\mathrm{r}}\right)\left(E / E_{\mathrm{c}}-1\right),
\end{aligned}
$$

where $E_{\mathrm{M}}$ is the maximal value of the field in the gap $\left(E_{\mathrm{M}}=V_{0} / d\right)$ and $E_{\mathrm{c}}$ is the critical electrical field, where a self-sustained discharge exists in the gap. In this case, i.e., at $E \geq E_{\mathrm{c}}$, the rate of carrier generation is not smaller than the rate of their recombination. The irradiation of the semiconductor decreases its resistance, thus enhancing the gap voltage and leading to increased radiation from the glow discharge. The interaction with capacitances of the system provokes the oscillatory behavior (spikes). Equations (1), (2) describe this behavior. The values of $\tau_{\mathrm{M}}$ can be calculated from the following equation:

$$
\tau_{\mathrm{M}}=\left(\varepsilon_{1} \varepsilon_{0} / \sigma_{1}\right)\left(1+C_{2} / C_{1}\right)
$$

with $C_{1}, C_{2}$ - capacitances of semiconductor plate and discharge gap, respectively; $\sigma_{1}, \varepsilon_{1}$ - conductivity and relative dielectric constant of the photodetector material, respectively and $\varepsilon_{0}$ - dielectric constant of vacuum. The sign on the right-hand side of Eq. (2) does not depend on the concentration of free carriers. This assumption is valid if the spatially homogeneous current density distribution in the gap can be described by the Townsend discharge mode [23]. The first part of the right-hand side in Eq. (1) describes the charging of the gap capacity $C_{2}$ by the voltage source $V_{0}$ through the semiconductor resistance. This process also depends on the semiconductor capacity $C_{1}$. The second part describes the discharging of the gap capacity due to free carrier in the gap [24]. Along most parts of the gas discharge gap, the ionic current exceeds the electron current. High electron mobility $\mu$ means high electron current $[25,26]$ :

$$
j_{\mathrm{e}} / j_{\mathrm{i}}=e N e \mu E \text {. }
$$

Consequently $j_{\mathrm{e}} / j_{\mathrm{i}}=\gamma<1$, this is the reason for the prevalence of the ion current in the cathode region of the glow discharge.

In the case of a dark Townsend discharge practically all the space is positively charged, with a low value of volume charge due to smallness of $j$ (current density) and $n_{+}$(number density of positive ions), and the field in the gap can be taken to be approximately constant as before [23]. From the criterion for the existence of the Townsend discharge mode [23] it can be easily verified that we have this kind of gas discharge in a thin gap gas discharge system. The criterion for a Townsend 
discharge mode will even be satisfied for current densities that are a few orders of magnitude higher than the current densities observed in the nonillumination system cell. In the Townsend discharge mode, the voltage drop across the gap does not depend on the current density. Therefore the curve actually demonstrates the CVC of the semiconductor, which has been moved from the coordinate origin along the voltage axis by $U_{\mathrm{B}}$ (more detailed information about the Townsend discharge will be given later).

A previous study of a SGDS with a SI GaAs cathode [15] revealed that some destabilization there commonly gives rise to spatially homogeneous oscillations. The properties of this oscillatory instability in the prebreakdown stage are mainly determined by the density of electric current. Two representative time dependences of the current behaviors under pressure 52 Torr at a different feeding voltage $V_{0}$, in darkness and under a maximum illumination intensities of light $L_{2}$, are shown in Fig. 3a,b.

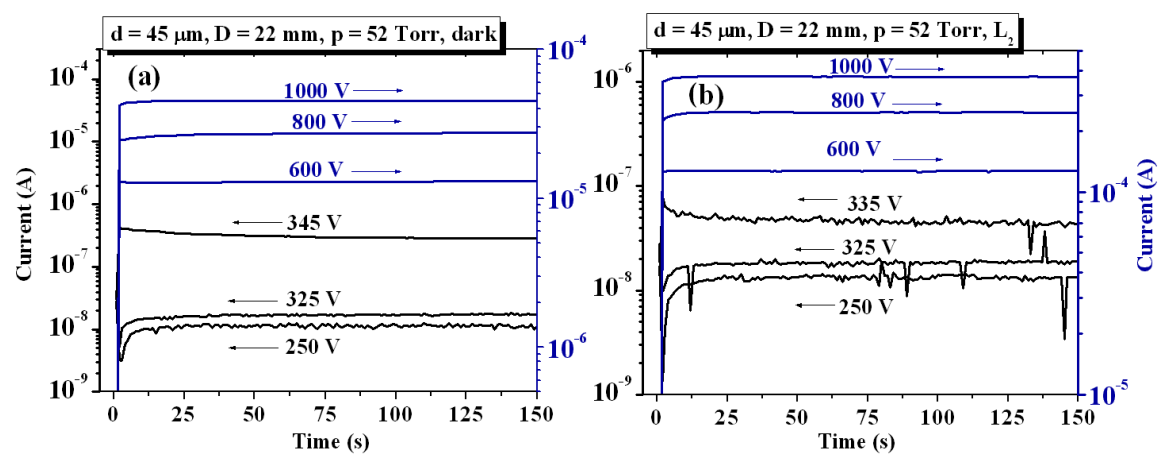

Fig. 3. Time dependence of current behavior in the SGDS (a) in darkness and (b) under maximum illumination intensities $L_{2}$, and different feeding voltages $V_{0}$ : for $V_{0}<V_{\mathrm{B}}$ (i.e. curves for $V_{0}=250$ and $325 \mathrm{~V}$ ); curves at $335 \mathrm{~V}$ and $345 \mathrm{~V}$ correspond to $V_{\mathrm{B}}$; for $V_{0}>V_{\mathrm{B}}$ (i.e. curves for $V_{0}=600,800$ and $1000 \mathrm{~V}$ ). The system parameters are $p=52$ Torr, $D=22 \mathrm{~mm}, d=45 \mu \mathrm{m}$.

The lowest two curves (i.e. for $V_{0}=250 \mathrm{~V}$ and $325 \mathrm{~V}$ ) oscillate with relatively small amplitudes of the prebreakdown currents. Besides, the third curves in both plots correspond to the breakdown voltage $\left(V_{B}=335 \mathrm{~V}\right.$ and $\left.345 \mathrm{~V}\right)$, at which the oscillation of current tends to be stable. The upper three curves correspond to voltages $V_{0}=600 \mathrm{~V}, 800 \mathrm{~V}$ and $1000 \mathrm{~V}$. It should be noted that monotonic steady state current behavior is observed for $V_{0}>V_{\mathrm{B}}$ up to $1000 \mathrm{~V}$. Meanwhile, as seen in Fig. 3a,b, the current values become stationary during the time range of $150 \mathrm{~s}$ and the value of the steady state current increases by increasing $V_{0}$. These observations, together with the data of the present work, show the existence of the very small oscillatory instability in the range of control parameters (i.e. for prebreakdown region, $V_{0}=250 \mathrm{~V}, 325 \mathrm{~V}$ and $345 \mathrm{~V}$ ) where the discharge gap may 
play an active role. Magnitudes of the steady state current and DLE established after some transient phase of the kinetics are determined both by the amplitude of the feeding voltage and by the value of resistance of the GaAs photodetector.

At this point, we assume that a homogeneous stationary Townsend discharge [27] is established in the SGDS. From the physical point of view, the most important feature of this kind of gas discharge is that space charge effects inside the gap are small and do not cause a distortion in the electric field between the electrodes. Another characteristic property is the homogeneous distribution of the current density perpendicular to the current flow. The DLE from the discharge is also homogeneous, while the wavelength of the DLE depends on the filled gas. The DLE intensity is proportional to discharge current. A local change of a semiconducting cathode resistance leads to a local change of the current and the DLE [18]. Hence, the principle of operation of the SGDS is based on some specific properties of the Townsend discharge. The voltage drop at the discharge gap for Townsend discharge mode is independent of the current. Therefore, the slope of the CVC provides the resistance of the GaAs photocathode. Then, the specific conductivity can be computed from the resistance and the geometric dimensions. We notice that the current density in the SGDS in the investigated parameter range does not exceed the limiting current for the existence of the Townsend discharge at given experimental conditions [4]. We remark that the feeding voltage $V_{0}$ is the sum of the voltage drops at the gas gap and at the semiconductor component. One of the characteristic features of the Townsend discharge is the constancy of the voltage drop $V_{\mathrm{B}}$ at the discharge domain while current varies. Figure 4 shows some examples of the stationary CVCs measured in the absence (see Fig. 1a) and in the presence of a gas discharge region (see Fig. 1b). The inset in Fig. 4 shows the $N$-type NDC in the SI GaAs photodetector in the structure of type $a$ (i.e. without gas discharge region) in Fig. 1a. A comparison of the CVC curves behavior measured for both structures with the same SI GaAs photodetector shows that the presence of the gas discharge region does not significantly modify the character of the charge transport in the structure studied: the corresponding CVC curves are only shifted by a value corresponding to the potential drop $V_{\mathrm{B}}$ of the gas discharge initiation. At the same time, a change in the illumination intensity does not lead to a significant shift of the critical voltage $V_{\text {cr }}$ (at which the current $I$ begins to drop by increasing $V_{0}$ ). One can conclude from the observed data that the electrical instabilities appear and disappear as soon as the voltage drop at the semiconductor is of certain critical values, which are constant for the chosen GaAs photodetector.

Thereby, we suppose that properties of the semiconductor are responsible for the appearance of the observed current instability and filamentation pattern. To prove this assumption, we have measured the CVC of the GaAs-layer without gas discharge. To perform the measurement, both surfaces of the semiconductor wafer have been coated with a thin Ni film. An example of data is shown in 


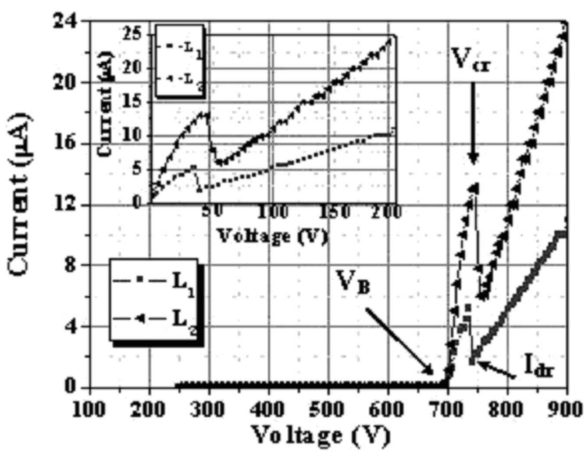

Fig. 4. $N$-shaped CVCs of the system including the GaAs photodetector with and without (see the inset) the gas-filled gap under weak and maximum illumination intensities of light $L_{1}$ and $L_{2}$, respectively. The material for the detector is the same that is used to obtain the data shown in the inset. The system parameters are $p=100$ Torr, $D=12 \mathrm{~mm}, d=323 \mu \mathrm{m}$.

Fig. 4. It is clear that the CVC of GaAs becomes strongly nonlinear when the voltage drop at the semiconductor $V_{\mathrm{s}}$ is kept around 40-50 V (see the inset in Fig. 4). The CVC of the Townsend discharge shows that the voltage drop at the gas $V_{\mathrm{g}}$ remains to be $700 \mathrm{~V}$ while the supply voltage $V_{0}$ changes from 250 to $900 \mathrm{~V}$. Thus, the nonlinear properties of GaAs start to play a role if the supply voltage $V_{0}=$ $V_{\mathrm{s}}+V_{\mathrm{g}}$ reaches the values of $700-750 \mathrm{~V}$. Thus, the current instabilities occur when the voltage drop at the gas discharge is constant and the semiconductor operates in the nonlinear regime. They occur due to the nonlinearity of the semiconductor cathode, while the gas discharge serves to visualize transport processes in GaAs. The effect has been interpreted in the cited work as being due to the electrical domain instability in the semiconductor electrode caused by the $N$-type CVC of the electrode [14]. This kind of transport nonlinearity is known to be responsible for propagating high electric-field domains in SI GaAs (see, e.g., [14]). When the voltage $V_{0}$ approaches to the threshold level $V_{\text {cr }}$ from below, the current $I$ in the structures exhibits oscillations of a relatively small amplitude. Such a behavior of SI GaAs samples has been observed in a previous review (see, e.g., recent review [23] devoted to the problem of domain instability in SI GaAs). Therefore, the $N$-type CVC in the SGDS with GaAs photodetector are in good agreement with results of other authors [14, 28].

For $V_{0} \geq V_{\text {cr }}$, the current usually oscillates in a broad range of voltages and both regular and irregular variations are encountered. At the same time, by increasing $V_{0}$, the current starts to drop to a value which is called current drop, $I_{\mathrm{dr}}$ (see the arrow with $I_{\mathrm{dr}}$ ). It is shown that the discharge gap played a passive role under the appropriate experimental conditions and is not responsible for the appearance of the current instability. 
Figure 2a shows the CVCs of the SGDS in parallel-plane geometry with different conductivity $\sigma$ of the SI GaAs photodetector, which is varied by its uniform illumination through the semitransparent Ni-contact. The CVCs allow us to determine the cell parameters: (1) breakdown voltage $V_{\mathrm{B}} ;(2)$ variation of conductivity $\sigma$ (or resistivity $\rho$ ) of the semiconducting photodetector at different illumination intensities $L$ (i.e. change of $\sigma=\partial J / \partial V$, or $\rho=\partial V / \partial J$ where $J$ is the current density). Figure 2a also shows that the CVCs are very close to a linear curve if $V>V_{\mathrm{B}}$ is satisfied.

As demonstrated in Fig. 2a, the potential drop across the discharge gap in this range is nearly constant and the electric field increases only on the SI GaAs photodetector [29]. The curves $\rho_{1}, \rho_{2}$ and $\rho_{3}$ in Fig. 2a represent the CVC (i.e. in darkness and under weak and maximum illumination intensities of light $L_{1}$ and $L_{2}$, respectively) when the resistivity of the SI GaAs photodetector is decreased from $\rho_{1}=1.3 \times 10^{8} \Omega \mathrm{cm}$ to $\rho_{3}=4 \times 10^{7} \Omega \mathrm{cm}$ through illumination. Moreover, the shape of the CVC in a system depends on the voltage increase in the power supply and the illumination intensity $[19,30]$. It should be noted that CVCs of SI GaAs photodetector show ohmic behavior for $d=143 \mu \mathrm{m}$. A further increase in the electrode separation $d$ beyond a certain threshold value $(d=323 \mu \mathrm{m})$ leads to the destabilization to a ohmic behavior of CVCs of SI GaAs (see Fig. 2b).

Thus we claim this specific $d$ value as a critical parameter in order to classify the dynamics of our system, because beyond this critical electrode distance $d$ (i.e. between $323-525 \mu \mathrm{m}$ ) we observed the above-mentioned pronounced $N$-like shape oscillatory behavior in currents which yields to an oscillatory bifurcation from a stationary state (also see Fig. 5). Inhomogeneous SI GaAs photodetectors with the current flow localized in small regions of active electrode area cause the observed variations in peak voltages, multiple NDC regions, peak spreading and exponentially rising current densities $j$.
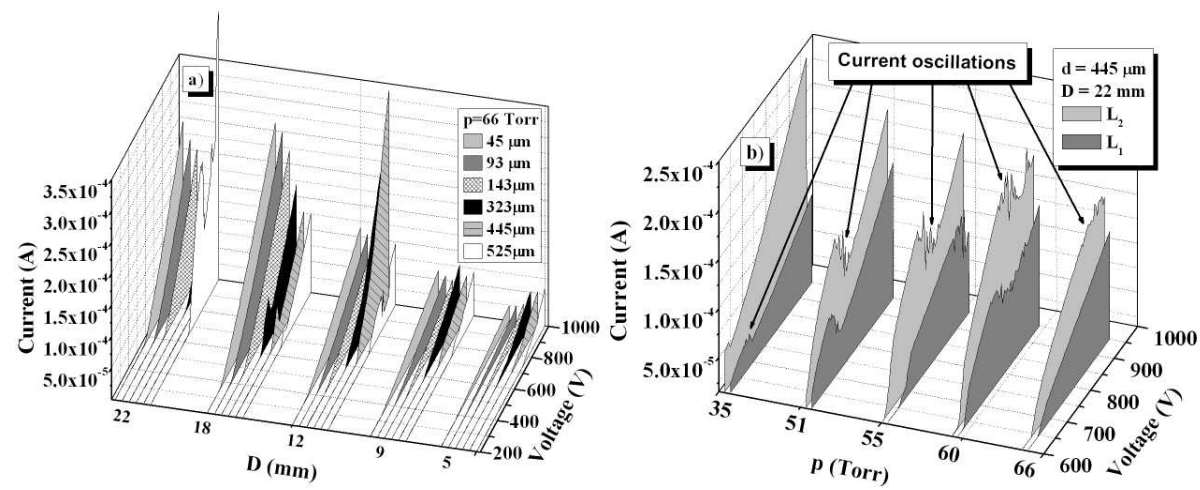

Fig. 5. (a) CVCs for different diameter $D$ of detector areas under maximum illumination intensity of light $L_{2}$. Gas pressure $p=66$ Torr. (b) $N$-shaped CVCs with oscillations in the case of $D=22 \mathrm{~mm}$ and $d=445 \mathrm{~mm}$ for different pressures. 
Curves in Fig. 5a represent the CVCs under maximum illumination intensity of light $L_{2}$, for different diameter $D$ of detector areas. As seen in Fig. 5, the range of current oscillations depends on the diameters $D$.

Considering these figures for the SGDS with different diameters $D$ of the detector areas, one can note the following: (a) for small values of the diameters $D$ (e.g. $D=9 \mathrm{~mm}$ ) the current oscillations are observed at $1.0 \times 10^{-5} \mathrm{~A}$ at first; (b) for large values of the diameters $D$ (e.g. $D=22 \mathrm{~mm}$ ) the current oscillations start at approximately $1 \times 10^{-4} \mathrm{~A}$.

The curves possess a clearly pronounced $N$-like shape which manifests them as apparent scatter in a dc measurement. Figure 5b demonstrates the behavior of the NDC and current oscillations more clearly in SI GaAs photodetector in the case of $D=22 \mathrm{~mm}$ and $d=445 \mathrm{~mm}$ for different pressures. If the CVC of the photodetector is a nonmonotonic one, instability develops in a SI GaAs, which may initiate nonlinear electrical characteristics in the device. Such a case is considered in this work. Our system shows a rich variety of instabilities by varying experimental parameters (thickness, $D$ and $\sigma$ of the photodetector material, gas pressure, etc.). The effect of pressure on CVCs for different diameters $D$ is shown in Fig. 6.



Fig. 6. CVCs of the SGDS with respect to pressure $p$ for different diameters $D$ of photodetector in darkness: (a) $D=9 \mathrm{~mm}$; (b) $D=12 \mathrm{~mm}$; (c) $D=18 \mathrm{~mm}$. The $525 \mu \mathrm{m}$ thick discharge gap is filled with air.

The most stable current behavior is seen in Fig. 6b since the fluctuations in current values are minimal. It indicates that the effect of diameter $D$ on the stability of system is vital since the fluctuations in Fig. $6 \mathrm{~b}$ (in the case of $D=$ $12 \mathrm{~mm}$ ) decay mostly. Another vital system variable on the current stability is the pressure $p$ since the patterns taken at lower $p$ are relatively more stable (see the first and fourth curves from left hand side in Fig. 6). Besides, the third parameter $V_{0}$ has also considerable effect. Let us note that the current values become relatively stable for higher $V_{0}$ values. All those effects take us to a conclusion about the current stability that the three experimental parameters, 
namely $p, D$, and $V_{0}$ play a complex and vital role and this phenomenon on the current stability has to be investigated for lower increment rates with higher parameter sensitivities. We estimate that a stability region should exist as a function of $p, V_{0}$ and $D$. Current oscillations of large amplitudes in such materials are related to the dynamics of electrical domains (generation, motion along the sample, and damping at the contacts) [14]. Under these conditions, instabilities of electrical current observed in the device are initiated by instabilities that occur in the GaAs photodetector. Just this concept has been put forward in papers (Ref. [31] and Ref. [32]) in order to interpret the occurrence of self-organization phenomena in SDGS systems with a SI GaAs cathode.

For further step, the electrical instability of the GaAs photodetector in the SDGS is analyzed by using both the current and DLE data as shown in Fig. 7a and b, respectively. (This structure is the basic component of the IR image converter; for details see, e.g., [19].)

Three typical cases, for darkness and under a weak $L_{1}$ and a maximum $L_{2}$ illumination of the detector, are represented in Fig. 7a. At the same time, Fig. 7a shows the change in the CVC of the structure for different resistance values $R$. Clearly, a reduction at the resistance of the photodetector shifts the threshold voltage where the current filaments are observed.

During the experiments on the emission properties of this kind of system, it is necessary to observe the gas brightness in the gap with high spatial and temporal resolution. Therefore, the DLE intensity is recorded simultaneously with the CVCs. For a thin discharge gap of the cell, the proportionality between the gas brightness and the current density $j$ can be observed in a broad range of $j$. Figure 7b shows the corresponding DLEs for the same structure with the SI GaAs photodetector. In this figure, electrical instabilities become more sensitive in the DLE oscillations (i.e. see the oscillations in Fig. 7b for dark). Therefore the DLE can demonstrate the current instabilities in SI GaAs detector much clearly. At the same time, it creates additional limitations on the resolution and determines threshold sensitivity for the system. When the current oscillations are observed in a type $b$ structure (i.e. with gas discharge region), the resolution $(\approx 8$ pairs of lines per millimeter) and stable operation of the system are disturbed. Detailed information regarding to time dependence of current fluctuations of large amplitude inside $N$-shape region of CVCs is represented in Fig. 7c. The values for 735 and $775 \mathrm{~V}$ in Fig. 7c corresponds to values at critical $V_{\text {cr }}$ and drop currents $I_{\mathrm{dr}}$ as stated in Fig. 4 for the case of $p=55$ Torr, $D=22 \mathrm{~mm}, d=445 \mu \mathrm{m}$. It is also clear that the current fluctuations of large amplitude inside $N$-shape region increase by feeding voltage. The above results take us to a conclusion that the state of the electric domain in transversely extended SI GaAs samples becomes unstable with respect to the transverse structure formation. This instability gives a rise to a complicated pattern of charge transfer in the semiconductor, thus revealing a new property of systems with the $N$-shaped CVC. 

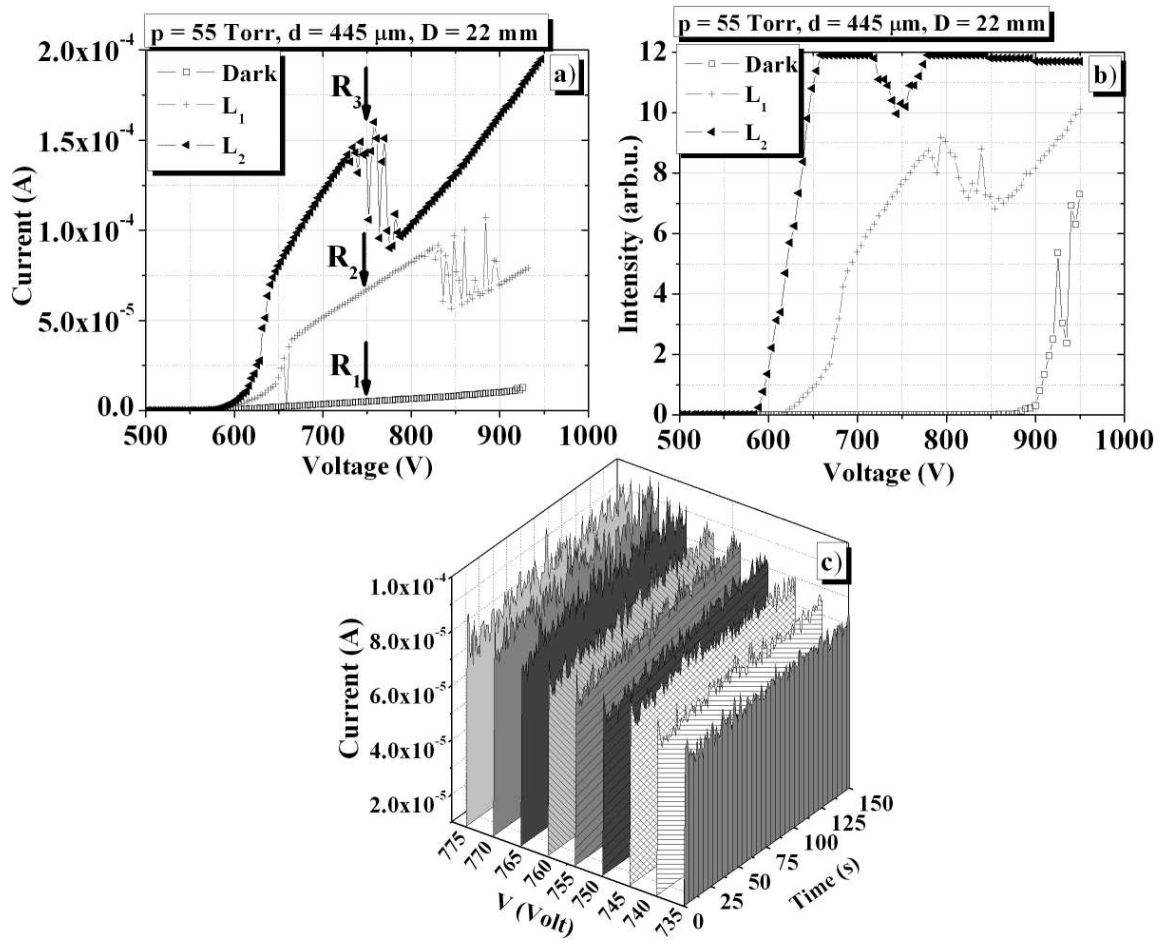

Fig. 7. (a) CVC of the SGDS for three resistances of the GaAs detector in darkness and under weak and maximum illumination intensities $L_{1}$ and $L_{2}$, respectively. The arrows (i.e. marked $R_{1}, R_{2}, R_{3}$ ) identify the values of $V_{\mathrm{th}}$, at which the filament pattern appears in the different resistance values of the photodetector active area. (b) The behavior of DLE in the SGDS for three resistances of the detector. System parameters are $p=55$ Torr, $D=22 \mathrm{~mm}, d=445 \mu \mathrm{m}$. (c) Time dependence of current oscillations (i.e. inside $N$-shape region of CVCs) in the structure with the gas discharge gap under maximum illumination intensity $L_{2}$ and different feeding voltages $V_{0}$.

A comparison of our findings for type $b$ structure and the data obtained from type $a$ structure by other authors for the SI GaAs photocathode $[31,33]$ shows that the presence of the gas discharge region does not significantly modify the character of the charge transport in our system. When photocathode with linear CVCs is used in the type $b$ structure, a spatially uniform distribution of the current density stays stable up to fairly high values of $j$. However, we have also investigated the filamentation formation in the type $b$ structure in the case the photocathode exhibits NDC. This has been achieved by a corresponding setting of the discharge cell parameters. In this case a spatially nonuniform distribution of the current density is observed frequently even at low values of $j\left(<1 \mathrm{~mA} / \mathrm{cm}^{2}\right)$. Such nonuniform distribution is easily visualized from the distribution of the intensity of the DLE. Two-dimensional (2D) pattern of DLE in the SGDS has been 
presented in Fig. 8. At the indicated conditions, the low-current gas discharge does not exhibit any pattern formation by itself and is characterized by a low temporal and spatial noise [34]. As seen in Fig. 8, dark regions correspond to a low discharge current, and hence, to a low conductivity of the IR photodetector plate. Thus, the conductance inhomogeneities transform to DLE inhomogeneities. The observed gas DLE pattern of detector plate allows one to assert that the stability disturbance regions are those of accumulation of inhomogeneities with a low conductivity.
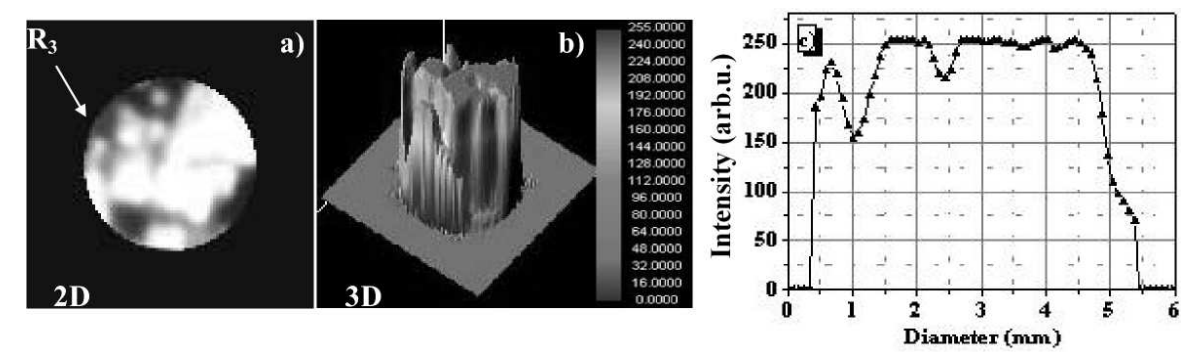

Fig. 8. The (a) 2D, (b) 3D filament patterns in gray level units and (c) variations of DLE intensity profiles crossing the active area of $3 \mathrm{D}$ surface pattern for resistance $R_{3}$ of the photodetector. Horizontal size of active area of the photodetector is $6 \mathrm{~mm}$.

Due to realize the quantitative analysis of DLEs, determination of light and dark regions of $2 \mathrm{D}$ pattern of the GaAs photodetector plate is required. Hence, 2D pattern of the GaAs photodetector is transformed into a 3D form. In the case of GaAs photodetectors, it is found that current filaments i.e. localized regions with a high current density appear in these samples. The observed current filamentation could give rise to a complex space-time behavior of the current distribution. However, the above-mentioned effect is usually accompanied by the formation of steady-state filaments. Visual observations indicate that the system exhibits spatially nonuniform states of the current involving formation of the multiple highcontrast filaments of the current in the region of these oscillations (i.e. marked $R_{1}, R_{2}, R_{3}$ in Fig. 7a). The corresponding filament pattern is depicted in Fig. 8 for resistance $R_{3}$, where the filament formation is observed. The essential feature of this pattern formation is that electric current can interactively be controlled there, by the amplitude of the feeding voltage and by the intensity of IR light, which irradiates the photodetector. Thus governs its photoconductivity [35]. The experimental results provide that instability is not governed by the gas discharge gap (for more detailed information see [31]).

The typical 2D and 3D examples of a DLE of filamentation pattern with resistance value $R_{3}$ of the photodetector active area in Fig. 7 is shown in Fig. 8. Typical dimensions of the filaments are $\approx 0.3-0.4 \mathrm{~mm}$. The threshold nature of the appearance of the filaments and the $N$-shaped CVC are evidences of the development of the instability in GaAs photodetector. 


\section{Conclusion}

Nonlinear electrical phenomena in a planar dc-driven SGDS with SI GaAs photodetectors are studied. The results demonstrate that the nonlinearity of the electrical conduction process is accompanied in SI GaAs photodetectors by the formation of high-contrast, spatially nonuniform distributions of the current. Such distributions include regions with a locally high current density (i.e. current filaments). SI GaAs photodetector, biased with dc voltages larger than a fixed critical voltage, shows oscillations in the current due to the presence of a high-field domain, which forms at the photocathode, propagates in the bulk, and disappears at the anode. A comparative study of the steady-state properties of two semiconductor structures (in one of them the anode is a gas discharge region and in the other it is an evaporated metal electrode) demonstrates that the instability of a spatially uniform distribution of the current observed in the structure with a discharge gap is due to the effects which take place in the photodetector. It is shown that under the suitable experimental conditions the discharge gap plays only a passive role and is not responsible for the appearance of the instability. It should also be noted that the dynamic and spatially inhomogeneous structures in the current distributions may negatively affect the characteristics of instruments based on SI GaAs photodetector. It is of importance to have knowledge in peculiarities of operation of these non-linear systems, in order to provide their stable and controllable operation. The experimental data presented in this work allows explaining the physical nature of phenomenon of the instabilities in SI GaAs photodetector and it is possible to choose optimum conditions to improve the output characteristics of this device in order to study the electrical instabilities in high resistivity IR photodetector plates.

\section{Acknowledgments}

This work is supported by Gazi University BAP research projects 05/2008-13 and $05 / 2008-20$.

\section{References}

[1] L.M. Portsel, Y.A. Astrov, I. Reimann, E. Ammelt, H. Purwins, J. Appl. Phys. 85, 3960 (1999).

[2] A.Kh. Zeinally, N.N. Lebedeva, L.G. Paritskii, B.G. Salamov, J. Photogr. Sci. 39, 114 (1991).

[3] N.N. Lebedeva, B.G. Salamov, V.I. Orbukh, V.M. Nagiev, Instrum. Exp. Tech. 37, 642 (1994)

[4] Y.P. Rayzer, Gas Discharge Physics, Springer, Berlin 1991, p. 126.

[5] H. Haken, Synergetics, Springer, Berlin 1978.

[6] A.V. Gorbatyuk, P.B. Rodin, Z. Phys. B, Condens. Matter 104, 45 (1997).

[7] B.G. Salamov, Y. Ciftci, K. Colakoglu, IEEE Trans. Plasma Sci. 32, 2093 (2004). 
[8] G.L. Lippi, P.A. Porta, L.M. Hoffer, H. Grassi, Phys. Rev. E 59, R32 (1999).

[9] Proc. 4th Int. Workshop on GaAs Detectors and Related Compounds, Eds. S. d'Auria, K.M. Smith, Nucl. Instrum. Methods Phys. Res. A 395, 1 (1997).

[10] A. Cola, L. Reggiani, L. Vasanelli, Semicond. Sci. Technol. 12, 1358 (1997).

[11] K. Berwick, M.R. Brozel, C.M. Buttar, M. Cowperthwaite, P. Sellin, Y. Hou, Mater. Sci. Eng. 28, 485 (1994).

[12] M. Kiyama, M. Yamada, M. Tatsumi, Eur. Phys. J. Appl. Phys. 27, 185 (2004).

[13] H.C. Ellin, A. Grunnet-Jepsen, L. Solymar, Appl. Phys. Lett. 65, 353 (1994).

[14] A. Neumann, J. Appl. Phys. 90, 15 (2001).

[15] H. Rajbenbach, J.M. Verdiell, J.P. Huignard, Appl. Phys. Lett. 53, 541 (1988).

[16] H.Y. Kurt, B.G. Salamov, T.S. Mammadov, Cryst. Res. Technol. 40, 1160 (2005).

[17] O. Godoy-Cabrera, J.S. Benitez-Read, R. Lopezcallejas, J. Pacheco-Sotelo, Int. J. Electron. 87, 361 (2000).

[18] B.G. Salamov, K. Çolakoğlu, Ş. Altındal, Infrared Phys. Technol. 36, 661 (1995).

[19] B.G. Salamov, S. Buyukakkas, M. Ozer, K. Colakoglu, Eur. Phys. J. Appl. Phys. 2, 275 (1998).

[20] R.S. Dhariwal, J.M. Torres, M.P.Y. Desmulliez, IEE Proceedings-Sci. Measurement Technol. 147, 261 (2000).

[21] H.Y. Kurt, B.G. Salamov, J. Phys. D, Appl. Phys. 36, 1987 (2003).

[22] V.N. Melekhin, N.Yu. Naumov, Sov. Phys.-Tech. Phys. 29, 888 (1984).

[23] N.N. Lebedeva, B.G. Salamov, B.G. Akinoglu, K.R. Allakhverdiev, J. Phys. D, Appl. Phys. 27, 1229 (1994).

[24] M.A. Guisinow, R.A. Gerber, J.B. Gerardo, Phys. Rev. Lett. 25, 1248 (1970).

[25] Ch. Radehaus, T. Dirksmeyer, H. Willebrand, H.G. Purwins, Phys. Lett. A 125, 92 (1987).

[26] H. Willebrand, T. Hunteler, F. Niedernostheide, R. Dohmen, H.G. Purwins, Phys. Rev. A 45, 8766 (1992).

[27] Y.P. Rayzer, Gas Discharge Physics, Nauka, Moscow 1987, p. 360 (in Russian).

[28] F. Piazza, P.C.M. Christianen, J.C. Maan, Phys. Rev. B 55, 15591 (1997).

[29] B.G. Salamov, K. Çolakoğlu, Ş. Altındal, M. Özer, J. Phys. III 7, 927 (1997).

[30] N.N. Lebedeva, V.I. Orbukh, B.G. Salamov, J. Phys. III France 6, 797 (1996).

[31] Y.A. Astrov, H.G. Purwins, Tech. Phys. Lett. 28, 910 (2002).

[32] E.L. Gurevich, A.S. Moskalenko, A.L. Zanin, Y.A. Astrov, H.G. Purwins, Phys. Lett. A 307, 299 (2003).

[33] L.D. Tsendin, Encyclopedia of Low-Temperature Plasma, Nauka, Moscow 2000, p. 16.

[34] V.M. Marchenko, S. Matern, H.G. Purwins, Y.A. Astrov, L.M. Portsel, Proc. SPIE 1, 4669 (2002).

[35] B.G. Salamov, S. Altindal, M. Ozer, K. Colakoglu, E. Bulur, Eur. Phys. J. Appl. Phys. 2, 267 (1998). 